\title{
MRI Features of Temporalis Muscle Flap Versus Dermis-fat Graft After TMJ Discectomy
}

\author{
Fatma Wageeh Attya ${ }^{1}$, Moustafa Mohamed El-Sayed Taha ${ }^{2}$, Ahmed Mohamed Salah ${ }^{1}$, \\ Mahmoud El-Shamy ${ }^{3}$, Mohammed Abd El-Mageed Katamesh ${ }^{2}$, \\ Mohamed Galal Gamal El-Deen Beheiri ${ }^{4}$ \\ ${ }^{1}$ Departement of Maxillofacial Surgery, Ahmed Maher Teaching Hospital, Cairo, Egypt \\ ${ }^{2}$ Departement of Maxillofacial Surgery, Faculty of Dentistry, Ain Shams University, Cairo, Egypt \\ ${ }^{3}$ Departement of Radiology, Faculty of Medicine, Al-Azhar University, Cairo, Egypt \\ ${ }^{4}$ Departement of Oral and Maxillofacial Surgery, Faculty of Oral and Dental medicine, Cairo University, Giza, Egypt
}

Email address:

fatmawageeh@hotmail.com (F. W. Attya),moustafataha@asfd.asu.edu.eg (M. M. El-Sayed T.), ahmadsalah81@hotmail.com(A. M. Salah), elshamy.radio@gmail.com (M. El-Shamy),katamish72@yahoo.com (M. A. El-Mageed K.), galalbeheiri@gmail.com (M. G. G. El-Deen B.)

\section{To cite this article:}

Fatma Wageeh Attya, Moustafa Mohamed El-Sayed Taha, Ahmed Mohamed Salah, Mahmoud El-Shamy, Mohammed Abd El-Mageed Katamesh, Mohamed Galal Gamal El-Deen Beheiri. MRI Features of Temporalis Muscle Flap Versus Dermis-fat Graft After TMJ Discectomy. International Journal of Medical Imaging. Vol. 8, No. 4, 2020, pp. 89-92. doi: 10.11648/j.ijmi.20200804.14

Received: October 2, 2020; Accepted: November 2, 2020; Published: November 11, 2020

\begin{abstract}
The aim of this study was to radiologically evaluate dermis-fat graft and temporalis muscle flap as replacements after TMJ discectomy. Patients and methods: fourteen female patients were categorized into two equal groups (seven patients on each group,) group (A) and group (B). Group A underwent TMJ discectomy followed by interpositional muscle flap. Group $\mathrm{B}$ underwent TMJ discectomy followed by interpositional dermis-fat graft. Pre-operative MRI (T1, T2 and proton density) were obtained in addition to 3 months and 12 months post-operative MRI for comparison and follow up of the graft. Results: Temporalis muscle flaps were diagnosed in all group (A) patients (12 joints). The dermis-fat graft was detected within the operated joints or surrounding the condyle in three patients of group B while mostly resorbed in the other four patients after one year follow up. Conclusion: Discectomy and dermis-fat graft appears to be a successful surgical option regarding to the clinical parameters. However, summed degenerative changes depending on surgical operation should be retained. Temporalis muscle flap still the gold standard after TMJ menesictomy.
\end{abstract}

Keywords: Temporalis Muscle Flap, TMJ Discectomy, Dermis-Fat Graft

\section{Review of Literature}

Discectomy has been used as a surgical treatment for severe internal derangement of the temporomandibular joint (TMJ) for many years with good outcomes [1-3].

Unfortunately, discectomy without replacements results in significant regressive remodeling of the mandibular condyle. Attempts to help reduce this effect have been made with the use of inter positional grafts [4, 5]. Dermis-fat graft has previously been reported for use as an interpositional material following gap arthroplasty in the management of TMJ ankylosis in many studies [6, 7].

While the clinical outcomes appear favorable for the use of this material in the TMJ, the studies concerning fate of the dermis-fat graft within a functional joint space after discectomy are sparse. Temporalis muscle flap is one of the most used flaps in maxillofacial surgery owing to the volume and nature of tissue available, its rugged vascular supply, and the ease of procurement [8].

It has long been used to fill the space after gap arthroplasty since it was described in 1872, Verneuil described its use following gap arthroplasty of the temporomandibular joint (TMJ) for ankylosis.(adopted from Edwards \& Feinberg, 2003)(8). Its first use as replacements after discectomy was innovated by Feinberg and Larsen in 1989 [9]. 


\section{Aim of the Study}

The purpose this study was to investigate the radiological fate of dermis-fat graft and temporalis muscle flap within the TMJ after discectomy using (MRI).

\section{Patients}

\subsection{Selection Criteria}

Fourteen patients who had ADDwor based on MRI examination; were recruited for this study. All patients had initially presented with severely deformed and unsalvageable articular discs that resulted in persistent and intolerable pain and dysfunction of the TMJ, despite at least 6 months of conservative TMJ therapy involving occlusal splints, medication and physiotherapy (figure 1).

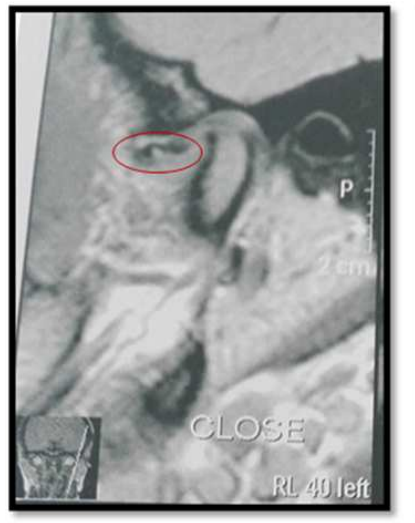

a

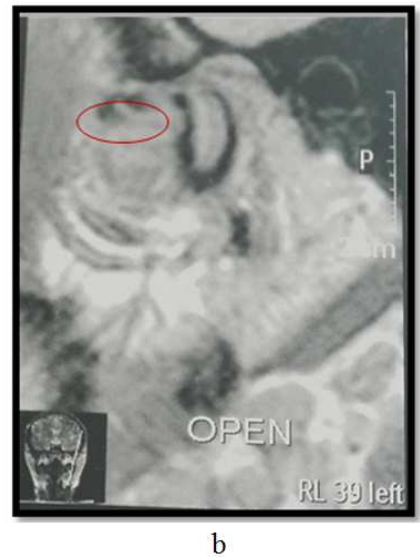

$\mathrm{b}$
Figure 1. Anterior disc displacement without reduction (a) closed view, (b) open view (PD TSE images).

\subsection{Study Design}

Patients were divided into two equal groups (each contain seven patients) control group (group A) and study group (group B). Patients had undergone TMJ discectomy through endaural approach with immediate placement of temporalis muscle flap (group A) or dermis-fat graft (group B) within the resultant joint space (figure 2).

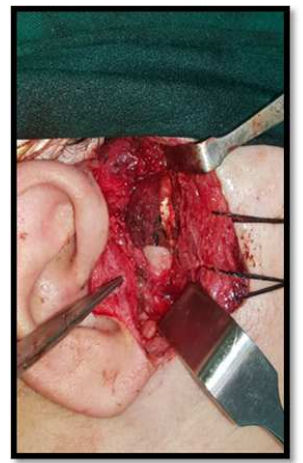

a

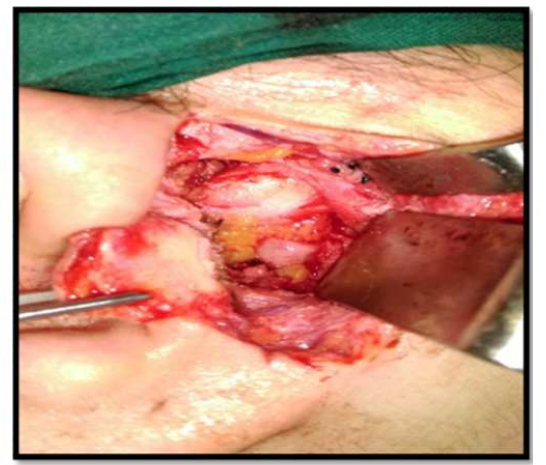

b
Figure 2. (a) Split thickness temporalis muscle flap sutured in place after discectomy in group A case. (b) dermis fat graft in place after discectomy in group B case.

\section{Methodology}

\subsection{Operative Details}

\subsubsection{Study Group}

In group B patients the dermis-fat grafts were procured from the lower abdomen through an elliptical incision that was closed primarily. The covering epidermis was removed by sharp dissection with a No. 15 scalpel blade and discarded.

The dermis-fat graft was trimmed and passively placed into the joint cavity to fill the whole space, and the surrounding capsule sutured to hold the graft in place.

\subsubsection{Control Group}

In group $\mathrm{A}$ the superior part of the endaural incision was extended two $\mathrm{cm}$ on most superior part of helix, $45^{\circ}$ to the zygomatic arch to avoid injury to the temporal branch of facial nerve. A finger-shaped flap extended as far superiorly was marked in the temporalis muscle to give proper length (about three-four $\mathrm{cm}$ ) to ensure adequate lining of the joint and avoid dead space formation.

The dissection was carried to the proper depth then the flap was extended to the level of the zygomatic arch. The flap was then rotated and sutured to the tissues medial, anterior and posterior to the disc through the already suspended sutures (2-0 resorbable suture; polyglactin 910) so that it covers the head of the condyle completely.

\subsection{Radiographic Evaluation}

All patients were scanned on a 1.5-TAvanto (Siemens Medical, Erlangen Germany) MR system using the standard 12-channel head coil. Patients were positioned comfortably and asked to keep their mouth closed for all but the last sequence, at which time a bite block was used to keep their mouths open as wide as comfortably possible. After appropriate localizers to identify the TMJ, the following sequences were acquired:

(1) closed mouth: (a) sagittal T1 \& proton density (PD) turbo spin echo (TSE), (b) axial T2 \& T1 TSE, (c) coronal T1 \& PD fat-saturated TSE, (2) open mouth:(b) sagittal PD TSE and T2 TSE.

The axial and coronal sequences were orthogonal to the body axis and centered on the TMJs. The sagittal sequences were positioned perpendicular to the head of the mandibular condyle and parallel to the ramus. The total examination time was under $30 \mathrm{~min}$ and all patients tolerated the examination well. The MR images obtained were examined and interpreted by an experienced MRI radiologist.

The grafts were evaluated regarding the following criteria:

Presence or absence of graft tissue within the joint space.

If present, nature of the tissue within and signal characteristics.

The joint was determined as the 120-degree arc extending from the mid body of the condylar head to the glenoid fossa in the sagittal and coronal planes. The joint was evaluated by $\mathrm{T} 1$ to assess secondary osteoarthritis changes and T2 to detect joint effusion. 


\section{Results}

There were 24 joints scanned in 14 patients: 12 left-side TMJs and 12 right-side TMJs. The radiological presence of graft tissue (whether temporalis muscle flap or dermis fat graft) was found in all 24 operated joints $(100 \%)$ that were scanned with MRI at 3 months post-operative. Dermis fat graft showed hyper intense signal at all pulse sequences while temporalis muscle flap showed isointense signal (figure 3).

The MRI scans taken at 12 months post-operative proved presence of temporalis muscle flap in all of the operated joints. Isointense signal of temporalis flap was found in five of the cases while the flap showed hypointense signal on the other two cases.
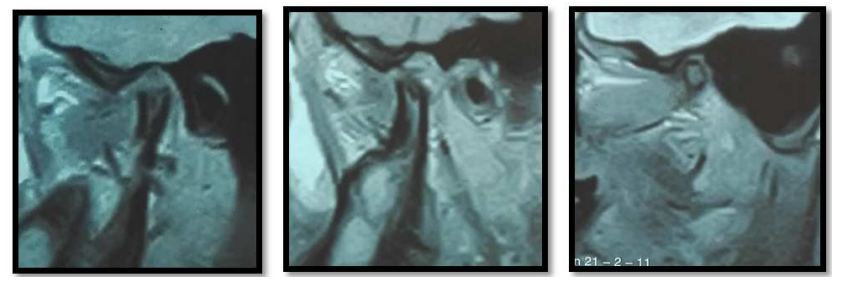

Figure 3. Sagittal oblique MRI PD image showing temporalis muscle flap filling the joint space from lateral to medial.

On the MRI taken 12 months post operatively the dermis fat grafts were almost resorbed on four cases ( 8 joints) while it was detected on the other 3 patients, reduced in size and was mainly lateral and posterior to the mandibular condyle (figure 4).

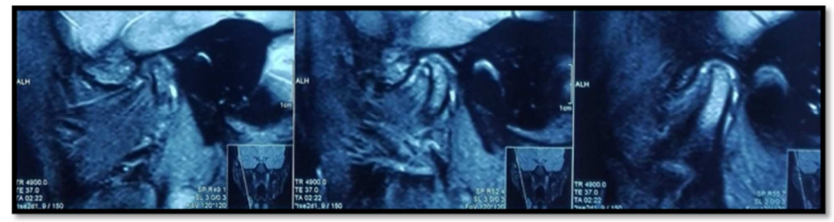

Figure 4. Sagittal oblique; T2 MRI showing dermis fat graft filling the joint space from lateral to medial.

\section{Discussion}

Current knowledge of TMJ pathologies largely depend on both the clinical presentations and MRI. Anterior disc displacement without reduction develop from alterations in the structural integrity of the condyle-disc complex. A definitive treatment that may be considered for such derangements is surgical correction.

surgery should be considered only when non-surgical therapy fails to adequately resolve the symptoms and/or progression of the disorder and it has been adequately determined (through correlation between clinical examination and MRI findings) that the source of pain is intracapsular structures [10].

Many authors support TMJ discectomy without replacements claiming that anew tissue would form between the condyle and fossa that will act as pseudo disc [11, 12]. A common finding in all discectomy patients reviewed is the significant changes in the condylar morphology of the operated joint [13] Clinical studies involving MRI, support the opinion that the radiographic changes of the condyle are adaptive rather than degenerative because the reduced symptoms do not correlate with the significant condylar changes seen following discectomy [13-15].

Whilst the literature clearly points to the long-term success of TMJ discectomy without replacement $[11,17,18]$ there is still concern about the crepitus and regressive remodeling that takes place in the condyle.

The temporalis muscle flap is the most popular interpositional flap for reasons of close proximity and ease of use. Moreover; the flap maintains its viability and functional movement during mandibular excursion [18]. In 1989, Feinberg and Larsen [9] reported on the technique for harvesting and in setting a pedicled temporalis muscle flap that was used in the present study.

Temporalis muscle flap examined using MRI 3 months postoperative showed isointense tissue filling the joint space similar in intensity to other muscles on the TMJ area on T1 enhanced MRI images while variable T2 signal intensity and varying degrees of enhancement relative to adjacent normal muscles.

The heterogeneity noted in T2 images is speculated to reflect the vascular disruption, neovascularization and muscle denervation.

On the second MRI exam performed 12 months later; temporalis muscle flap showed decreased volume and areas of hypo intensity supposed to be due to some degree of muscle fibrosis to compensate with the new function of lining a loaded joint.

In group B patients, MRI after 3 months post-operative showed hyper intense signal that is similar to fat in all the operated joints. After 12 months follow up From the investigation using MRI it was found that the grafted fat reduced in size to about $33 \%$ of the original size in three cases ( 6 joints) while nearly resorbed in the other four patients of group B ( 8 joints). The signal intensity of the grafted fat was lower than that of normal subcutaneous fat tissue.

The hypointense signal might indicate fibrosis of the graft in response to the joint function. The presence of dermis could not be discerned on the images obtained, which was probably due to the limitation of the imaging technology rather than absence of the dermis.

The most remarkable finding of this study was the survival of the dermis fat graft, which was seen in six of the operated joints. The fate of transplanted fat may be linked to adipose precursor cells which are distributed widely in connective tissues throughout the adult body [18]. Such cells in the surrounding connective tissue can proliferate and mature into fat cells depending on the micro-environment [18].

Intermittent compressive forces of the joint may act as a negative influence on the growth and maintenance joint of fat tissue within the joint space itself [18] and that might be the cause of early fat degeneration in four cases of group B who didn't stuck to postoperative instructions including eating 
hard food and not wearing the stabilization splint.

\section{Conclusion}

The survival of free dermis fat grafts implanted into bony cavities other than bone. The -implanted free fat dermis graft prevented ankylosis and acted as an efficient gap filling material.

Temporalis muscle flap can preserve adequate volume and strength to protect articulating surfaces after TMJ discectomy and still preferred as interpositional tissue after discectomy.

\section{References}

[1] Dimitroulis G, McCullough M\& Morrison W. Quality-of-Life Survey Comparing Patients Before and After Discectomy of the Temporomandibular Joint. Journal of Oral and Maxillofacial Surgery, 68 (1), 101-106, 2010.

[2] Eriksson L \& Westesson PL. Discectomy as an effective treatment for painful temporomandibular joint internal derangement: A 5-year clinical and radiographic follow-up. Journal of Oral and Maxillofacial Surgery, 59 (7), 750-758, 2001.

[3] Holmlund AB. Surgery for TMJ internal derangement. Evaluation of treatment outcome and criteria for success. International Journal of Oral and Maxillofacial Surgery, 22 (2), 75-77, 1993.

[4] Holmlund A, Lund B \& Weiner CK. Discectomy without replacement for the treatment of painful reciprocal clicking or catching and chronic closed lock of the temporomandibular joint: a clinical follow-up audit. British Journal of Oral and Maxillofacial Surgery, 51 (8), e211-e214, 2013.

[5] Ioannides C \& Freihofer HP. Replacement of the damaged interarticular disc of the TMJ. Journal of Cranio-MaxilloFacial Surgery: Official Publication of the European Association for Cranio-Maxillo-Facial Surgery, 16 (6), 273 $278,1988$.

[6] Mackay DR, Manders EK, Saggers GC, Schenden MJ \& Zaino R. The fate of dermal and dermal-fat grafts. Annals of Plastic Surgery, 31 (1), 42-46, 1993.

[7] Georgiade N, Altany F \& pickrell k. An experimental and clinical evaluation of autogenous dermal grafts used in the treatment of temporomandibular joint ankylosis. Plastic and Reconstructive Surgery (1946), 19 (4), 321-336, 1957.
[8] Edwards SP\&Feinberg SE. The temporalis muscle flap in contemporary oral and maxillofacial surgery. Oral and Maxillofacial Surgery Clinics of North America, 15 (4), 513535, 2003.

[9] Feinberg SE\& Larsen PE. The use of a pedicled temporalis muscle-pericranial flap for replacement of the TMJ disc: preliminary report. Journal of Oral and Maxillofacial Surgery: Official Journal of the American Association of Oral and Maxillofacial Surgeons, 47 (2), 142-146, 1989.

[10] Okeson JP. Management of Temporomandibular Disorders and Occlusion, 2007.

[11] Takaku S, Sano T \& Yoshida M. Long-term magnetic resonance imaging after temporomandibular joint discectomy without replacement. Journal of Oral and Maxillofacial Surgery, 58 (7), 739-745, 2000.

[12] Takaku S \& Toyoda T. Long-term evaluation of discectomy of the temporomandibular joint. Journal of Oral and Maxillofacial Surgery: Official Journal of the American Association of Oral and Maxillofacial Surgeons, 52 (7), 7226-8, 1994.

[13] Tolvanen M, Oikarinen VJ \& Wolf J. A 30-year follow-up study of temporomandibular joint meniscectomies: a report on five patients. The British Journal of Oral \& Maxillofacial.

[14] Dimitroulis G, Trost N \& Morrison W. The radiological fate of dermis-fat grafts in the human temporomandibular joint using magnetic resonance imaging. International Journal of Oral and Maxillofacial Surgery, 37 (3), 249-254, 2008.

[15] McKenna SJ. Discectomy for the treatment of internal derangements of the temporomandibular joint. Journal of Oral and Maxillofacial Surgery, 59 (9), 1051-1056, 2001.

[16] Politi M, Sembronio S, Robiony M, Costa F, Toro C \& Undt G. High condylectomy and disc repositioning compared to arthroscopic lysis, lavage, and capsular stretch for the treatment of chronic closed lock of the temporomandibular joint. Oral Surgery, Oral Medicine, Oral Pathology, Oral Radiology, and Endodontology, 103 (1), 27-33, 2007.

[17] Brusati R, Raffaini M, Sesenna E\& Bozzetti A. The temporalis muscle flap in temporo-mandibular joint surgery. Journal of Cranio-Maxillofacial Surgery, 18 (8), 352-358, 1990.

[18] N Kawaguchi, K Toriyama, E Nicodemou-Lena, K Inou, S Torii, Y Kitagawa. De novo adipogenesis in mice at the site of injection of basement membrane and basic fibroblast growth factor. Proc Natl Acad Sci U S A, 3; 95 (3): 1062-6, 1998 Feb. 